\title{
Fiber Scanning Array for 3 Dimensional Topographic Imaging
}

\author{
D. Barry Coyle, David L. Rabine, Demetrios Poulios*, J. Bryan Blair, Paul R Stysley, Richard Kay*, and \\ Greg Clarke*, Jack Bufton** \\ Code 554/694, NASA Goddard, Greenbelt MD \\ *Dept. of Physics, American University, Washington DC \\ **Global Science Tech, Greenbelt, MD \\ barry.coyle@nasa.gov
}

\begin{abstract}
We report on the design and development of a fiber optic scanning 3-D LIDAR employing a switched fiber array. This design distributes ns length laser pulses over a sample field, collects the return pulses, and assembles them into a 3-D image. This instrument is a reduced size version consisting of 35 beams, and will serve as a proof-ofprinciple demonstration for a planned 1000 beam instrument for Earth and planetary topographical missions.

OCIS codes: $060.2380,110-2350$
\end{abstract}

Wide-swath topographic mapping has been a key tool for observing the Earth's surface topography at local, regional, and global scales, and is of great importance for our ability to characterize, understand, and model the processes that shape the surface of our planet. A Mission Design Study conducted at NASA/GSFC concluded that an instrument design using 1,000 beams where each beam illuminates a $5 \mathrm{~m}$ spot on the ground would be sufficient to meet requirements for the Decadal Survey's Lidar in Space Technology (LIST) mission addressing these science goals. Such an instrument would be ideally created using a fiber optic architecture. We have elected to create a smaller proof-of-principle version that would be upgradable in all aspects of the design. This smaller version uses a 35 beam LIDAR based on a newly created 1x35 beam fiber switch from AdVR[1]. The design will also leverage "point-and-fire" technique for swath mapping employed by LVIS (Laser Vegetation and Ice Sensor) which uses a single receiver and detector as a method for covering large areas with maximum resolution, high reliability, high precision, and optimum data volume [2]. Performance expectations in accuracy, special coverage, and vertical precision will make this design easily adaptable to applications such as autonomous landing hazard avoidance (ALHAT) robotic imaging and servicing applications, and for use on topographical planetary targets such as Ceres.

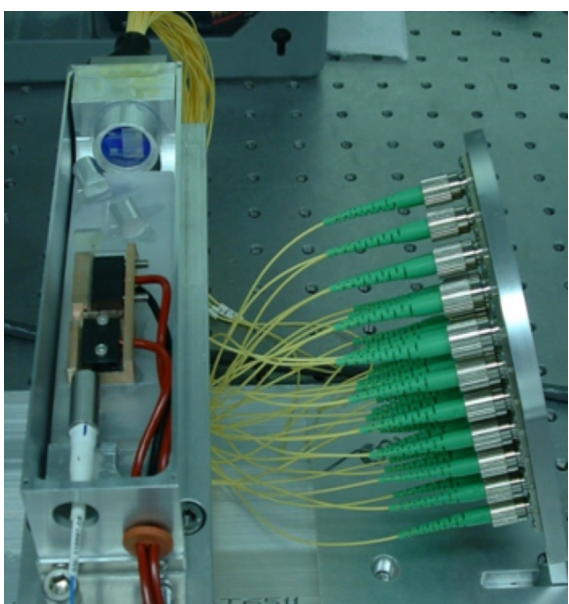

Figure 1(a): 1 x 35 electro-optic fiber switch developed under NASA's SBIR program.

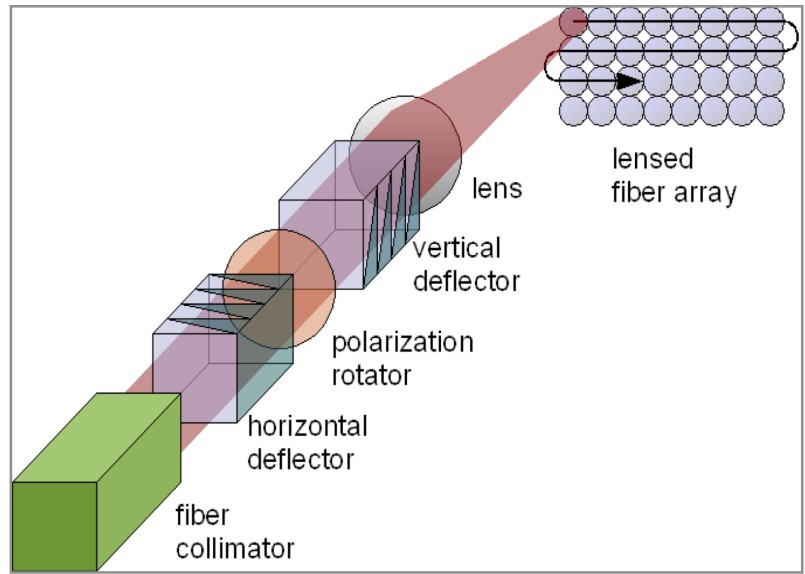

Figure 1(b): Block diagram showing operation of fiber switch. Poled MgO:LN crystals steer laser pulses into individual fibers with high speed and low optical loss. 
The 1 X 35 switch, referred to as the Fiber Switched Array (FSA) is shown in Figure 1a and its internal design shown in Figure 1b. The fiber collimator accepts a series of laser pulses and sends them through a series of two deflectors, $x$ and $y$. Each pulse is directed to a different optical fiber attached to the lensed fiber array. Each output fiber is thereby individually addressable. This switched array has been tested and works with sub ns pulses.

The switch will then be integrated into the instrument design shown in Figure 2. A fiber-coupled $1064 \mathrm{~nm}$ diode emitting short (ns) laser pulses serves as the seed source for a Yb:doped active fiber amplifier. The fiber amplifier assembly amplifies the picojoule-level seed pulses into the micro-joule range. After proper characterization and optical isolation, the output is coupled into the FSA input fiber.

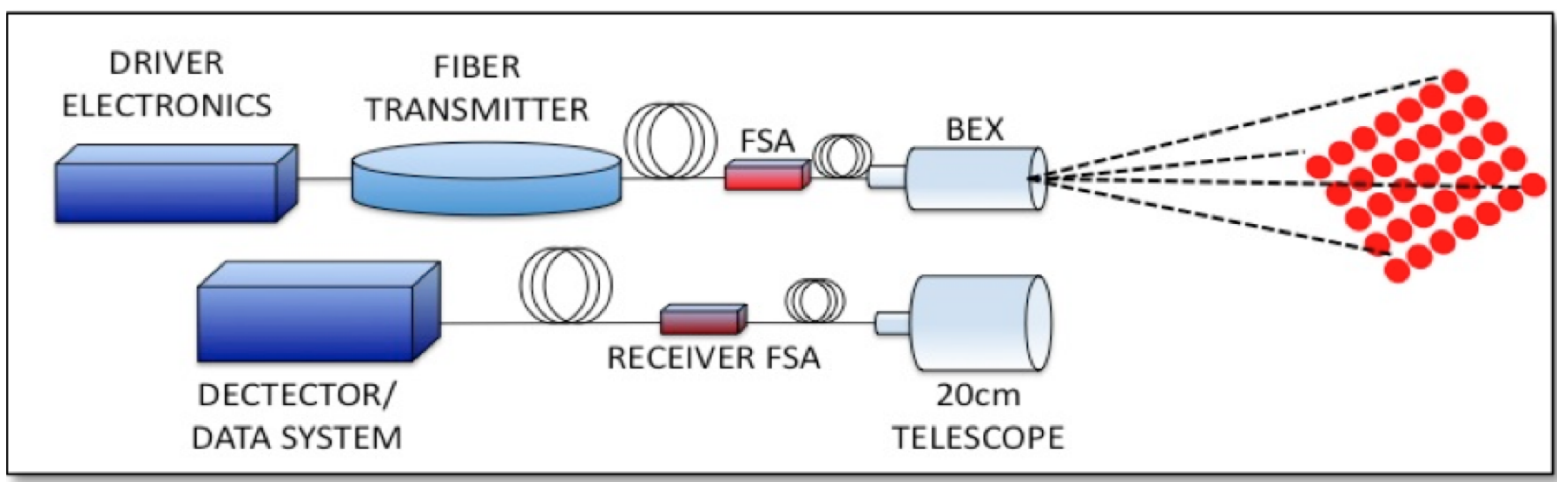

Figure 2: Block diagram of proposed LIST lidar system. The transmitter FSA traces out a programmable $5 \times 7$ scan pattern on a surface, while the receiver FSA rapidly directs the receive IFOV to observe the transmitter pixel.

This whole chain of optical pulse production and amplification lies within a fusion spliced line of fiber, and so is immune to contamination and is very thermally and mechanically stable. The larger amount of attention has been on the FSA drive design. The FSA operates with the use of a pair of $\mathrm{MgO}: \mathrm{LN}$ crystals in series, where each has an attached pair of high voltage electrodes. One crystal sweeps the transmitted beam in the $\mathrm{X}$ axis, where the $2^{\text {nd }}$ crystal sweeps the Y. A custom lenslet array lies on the focus of a positive lens after the $2^{\text {nd }}$ crystal. Each lenslet lies at a corresponding $\mathrm{X}$ and $\mathrm{Y}$ voltage for each crystal. Each crystal provides a full lenslet sweep in approximately $+/-300 \mathrm{~V}$, and a pair of pulsed HV, high speed power supplies drive each axis. Our main effort has involved developing the algorithm, circuitry, and software to drive these HV units to provide efficient, high speed, programmable optical switching among the 35 fibers. Because of the all-digital electro-optic design, the 35 channel fiber output parameters will be completely tunable in number of pulses per fiber, the order of sequential output fibers, the pulse energy, repetition rate as well. Although Figure 2 shows a corresponding FSA on the receiver, for this effort have simply designed a wide field of view telescope and broad area detector. By carefully time and channel tagging each emitted laser pulse, we can log each return signal from our laboratory target sample, (trees across the parking lot, for example) and generate 3D images. The design presented is readily scalable in power and number of beams employed. Once completed immediate plans would involve scaling up to a flight capable 1000 beam swath mapping system as required by missions such as LIST.

[1] AdvR Inc., 2310 University Way; Bldg. \#1-1, Bozeman, Montana 59715

[2] J.B. Blair, D. L. Rabine, and M.A. Hofton, "The Laser Vegetation Imaging Sensor: a medium-altitude, digitization-only, airborne laser altimeter for mapping vegetation and topography", ISPRS Journal of Photogrammetry and Remote Sensing, vol. 54, 115-122 (1999). 